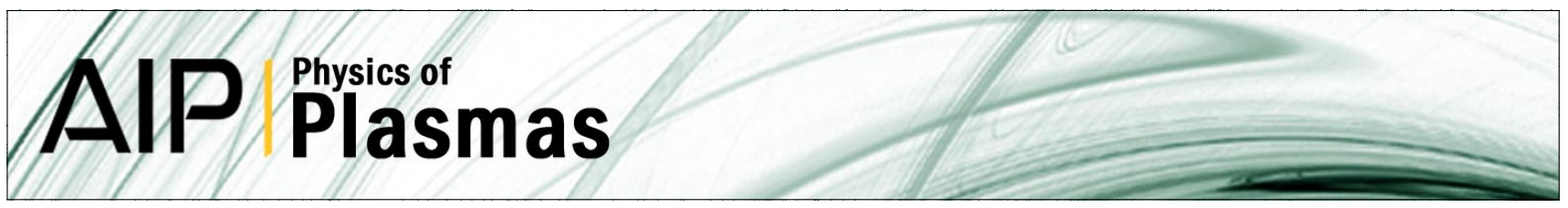

\title{
Turbulent regimes in the tokamak scrape-off layer
}

Annamaria Mosetto, Federico D. Halpern, Sébastien Jolliet, Joaquim Loizu, and Paolo Ricci

Citation: Phys. Plasmas 20, 092308 (2013); doi: 10.1063/1.4821597

View online: http://dx.doi.org/10.1063/1.4821597

View Table of Contents: http://pop.aip.org/resource/1/PHPAEN/v20/i9

Published by the AIP Publishing LLC.

\section{Additional information on Phys. Plasmas}

Journal Homepage: http://pop.aip.org/

Journal Information: http://pop.aip.org/about/about_the_journal

Top downloads: http://pop.aip.org/features/most_downloaded

Information for Authors: http://pop.aip.org/authors

\section{ADVERTISEMENT}

\section{AIP Applied Physics Letters}

\section{EXPLORE WHAT'S NEW IN APL}

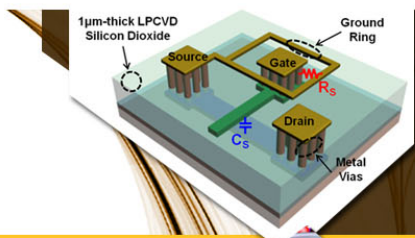

SURFACES AND INTERFACES

Focusing on physical, chemical, biological,

structural, optical, magnetic and electrical

properties of surfaces and interfaces, and more.. 


\title{
Turbulent regimes in the tokamak scrape-off layer
}

\author{
Annamaria Mosetto, ${ }^{\text {a) }}$ Federico D. Halpern, Sébastien Jolliet, Joaquim Loizu, \\ and Paolo Ricci \\ École Polytechnique Fédérale de Lausanne (EPFL), Centre de Recherches en Physique des Plasmas, \\ Association Euratom-Confédération Suisse, CH-1015 Lausanne, Switzerland
}

(Received 28 June 2013; accepted 16 August 2013; published online 25 September 2013)

The non-linear turbulent regimes in the tokamak scrape-off layer (SOL) are identified according to the linear instability responsible for the perpendicular transport. Four regions of the SOL operational parameters are determined where turbulence is driven by the inertial or resistive branches of the ballooning mode or of drift waves. The analysis, based on the linear electrostatic drift-reduced Braginskii equations, evaluates the pressure scale length selfconsistently from the balance between plasma losses at the vessel and perpendicular turbulent transport. The latter is estimated by assuming that turbulence saturation occurs due to a local flattening of the plasma gradients and associated removal of the linear instability drive; it is also shown that transport is led by the mode that maximizes the ratio of the linear growth to the poloidal wavenumber. The methodology used to identify the turbulent regimes is confirmed by the results of non-linear simulations of SOL turbulence. The identification of the turbulent regimes, the predicted pressure scale length, and the poloidal wavenumber of the leading mode are in reasonable agreement with non-linear simulation results. [http://dx.doi.org/10.1063/1.4821597]

\section{INTRODUCTION}

By governing the heat load on the plasma facing components, controlling the power and particle balance, and regulating the impurity dynamics, the scrape-off layer (SOL) is of fundamental importance to determine the performance of a tokamak. ${ }^{1}$ The plasma dynamics in this region results from the plasma outflow from the core, turbulent transport across the magnetic field lines, parallel flow, and losses at the limiter or divertor plates. The understanding of the interplay between those phenomena is necessary to optimally operate present and future tokamak devices.

A number of instabilities, driven by magnetic unfavourable curvature and plasma gradients, are possibly responsible of SOL plasma turbulence ${ }^{2-6}$ and different turbulent regimes have been experimentally identified. ${ }^{7}$ These regimes have also been identified by low-frequency, non-linear electromagnetic models. ${ }^{2,3,8-12}$ Among these instabilities, ballooning modes (BMs) and drift waves (DWs) are thought to play the most important role.

BMs are curvature driven instabilities destabilized when the plasma pressure gradient points in the same direction as the magnetic field line curvature, in the presence of finite resistivity, electron mass, or plasma $\beta \cdot{ }^{13-15} \mathrm{DWs}$ are caused by a pressure gradient and are destabilized by either finite electron mass or resistivity. ${ }^{16-18}$ The linear and nonlinear behaviors of these modes have been extensively

\footnotetext{
${ }^{\text {a) }}$ Author to whom correspondence should be addressed. Electronic mail: annamaria.mosetto@epfl.ch
}

studied. $^{2,3,8-11,13-27}$ Both BMs and DWs can be active in the SOL, but the knowledge of the conditions under which one or the other dominates is still lacking, despite the fact that this is essential to understand and predict the plasma dynamics in the SOL region. The goal of the present paper is to identify the SOL turbulent regimes, determining the driving instability, as a function of the SOL operational parameters.

In a previous work, ${ }^{28}$ we have presented a detailed description of the linear properties of the $\mathrm{BM}$ and $\mathrm{DW}$ modes, providing also a tool to identify the nature of the fastest growing linear modes, once the SOL pressure gradient length is known. This is a starting point of the present work, where we determine the instability dominating the non-linear plasma dynamics, i.e., the mode that leads to the major contribution to turbulent transport. Our analysis considers the pressure scale length as the self-consistent result of the interplay between plasma losses and turbulent transport, and that the mode dominating the non-linear plasma dynamics does not necessarily correspond to the fastest growing mode.

Our study is made possible by the investigation of the mechanisms leading to the saturation of the linearly unstable modes. ${ }^{29}$ For typical SOL parameters, the saturation is provided by the gradient removal mechanism, i.e., the saturation of the linear mode due to the non-linear flattening of the driving plasma gradients. The gradient removal theory provides an estimate of the plasma pressure scale length as a function of the SOL operational parameters, in quantitative good agreement with simulation ${ }^{29}$ and experimental results. ${ }^{30}$ It also allows us to identify the instability that dominates the non-linear dynamics. By studying the nature of 
this instability, we can determine the SOL turbulent regimes as a function of the SOL operational parameters, i.e., the safety factor, $q$, the magnetic shear, $\hat{s}$, the resistivity, $\nu$, and the ion to electron mass ratio, $m_{i} / m_{e}$. Our work concentrates on a relatively simple, circular, inner-wall limited configuration. Understanding a circular configuration is a departure point for studying more complicated geometries and regimes.

We also report on a set of non-linear simulations that support our methodology to identify the SOL turbulent regimes. The simulations are performed by using the GBS code, ${ }^{29}$ a flux-driven code that implements the drift-reduced Braginskii equations (see, e.g., Refs. 2 and 31) with a set of boundary conditions describing the magnetic pre-sheath entrance. ${ }^{32}$ The main feature of GBS is the capability of evolving fluctuations and background self-consistently, without imposing a fixed background gradient, and with no scale separation between fluctuations and equilibrium quantities. The code is therefore ideal to study the self-consistent formation of the plasma pressure gradient and, as a consequence, the SOL turbulent regimes as a function of the operational parameters.

The paper is organized as follows. After the Introduction, in Sec. II, we introduce the SOL plasma model used throughout our study. In Sec. III, we describe the non-linear saturation mechanism at play in the SOL, and we calculate the equilibrium pressure scale length depending on the SOL operational parameters. In Sec. IV, we describe the SOL turbulent regimes, and we present the investigation of the transitions among those. Section $\mathrm{V}$ is focused on the description of non-linear simulations carried out with the GBS code, supporting the methodology that we have previously described. Finally, we draw our conclusions in Sec. VI.

\section{THE MODEL}

Our study of plasma turbulence in the SOL is based on the two-fluid, electrostatic, non-linear, drift-reduced Braginskii equations. ${ }^{29}$ The fluid approach is justified by the high plasma collisionality in the SOL.

For the sake of simplicity, we consider $T_{i} \ll T_{e}$, since the fundamental properties of the dominant SOL instabilities, BMs, and DWs can be captured within a cold-ion model. The ion temperature gradient instability will be the subject of a forthcoming study and it is believed to be of secondary importance at the high resistivity characterizing the tokamak SOL. ${ }^{22}$ We also consider the electrostatic limit, neglecting the ideal branch of the BM. The role of the ideal $\mathrm{BM}$ in SOL turbulence is investigated in Ref. 30.

In the drift-reduced limit, we assume for the perpendicular velocities $\mathbf{V}_{\perp i}=\mathbf{V}_{E \times B}+\mathbf{V}_{p o l}$ and $\mathbf{V}_{\perp e}=\mathbf{V}_{E \times B}+\mathbf{V}_{* e}$, where $\mathbf{V}_{E \times B}=(-\nabla \phi \times \mathbf{b}) / B$ is the $\mathbf{E} \times \mathbf{B}$ drift velocity, $\mathbf{V}_{* e}=\mathbf{b} \times \nabla p_{e} /(e n B)$ is the electron diamagnetic drift velocity and $\mathbf{V}_{\text {pol }}$ is the ion polarization velocity (see, e.g., Ref. 33). The equations that describe the evolution of density, $n$, potential, $\phi$, electron parallel velocity, $V_{\| e}$, electron temperature, $T_{e}$, and ion parallel velocity, $V_{\| i}$, are

$$
\begin{aligned}
\frac{\partial n}{\partial t}= & -R[\phi, n]+2\left[\hat{C}\left(p_{e}\right)-n \hat{C}(\phi)\right]-\nabla_{\|}\left(n V_{\| e}\right) \\
& +S_{n}+D_{n}(n) \\
\frac{\partial \nabla_{\perp}^{2} \phi}{\partial t}= & -R\left[\phi, \nabla_{\perp}^{2} \phi\right]-V_{\| i} \nabla_{\|}\left(\nabla_{\perp}^{2} \phi\right)+\frac{2 \hat{C}\left(p_{e}\right)}{n}+\frac{1}{n} \nabla_{\|} j_{\|} \\
& +\frac{1}{3 n} \hat{C}\left(G_{i}\right)+D_{\omega}\left(\nabla_{\perp}^{2} \phi\right) \\
\frac{\partial V_{\| e}}{\partial t}= & -R\left[\phi, V_{\| e}\right]-V_{\| e} \nabla_{\|} V_{\| e}+D_{V_{\| e}}\left(V_{\| e}\right) \\
& +\frac{m_{i}}{m_{e}}\left(\nabla_{\|} \phi-\frac{1}{n} T_{e} \nabla_{\|} n-1.71 \nabla_{\|} T_{e}+\frac{\nu}{n} j_{\|}-\frac{2}{3 n} G_{e}\right) \\
\frac{\partial T_{e}}{\partial t}= & -R\left[\phi, T_{e}\right]+\frac{4}{3}\left[\frac{7}{2} T_{e} \hat{C}\left(T_{e}\right)+\frac{T_{e}^{2}}{n} \hat{C}(n)-T_{e} \hat{C}(\phi)\right] \\
& +\frac{2 T_{e}}{n} 0.71 \nabla_{\|} j_{\|}-\frac{2}{3} T_{e} \nabla_{\|} V_{\| e}-V_{\| e} \nabla_{\|} T_{e} \\
& +D_{T_{e}}\left(T_{e}\right)+S_{T_{e}} \\
\frac{\partial V_{\| i}}{\partial t}= & -R\left[\phi, V_{\| i}\right]-V_{\| i} \nabla_{\|} V_{\| i}-\frac{1}{n} \nabla_{\|} p_{e} \\
& -\frac{2}{3 n} \nabla_{\|} G_{i}+D_{V_{\| i}}\left(V_{\| i}\right),
\end{aligned}
$$

where $R$ is the tokamak major radius, $\nu=R e^{2} n_{0} /\left(c_{s 0} m_{i} \sigma_{\|}\right)$ is the normalized parallel resistivity, being $\sigma_{\|}=1.96$ $n_{0} e^{2} \tau_{e} / m_{e}$ the parallel Spitzer conductivity. The source terms $S_{n}$ and $S_{T_{e}}$ mimic the flow of plasma into the SOL through the last closed flux surface. The terms $D_{f}(f)$ represent small perpendicular diffusion added for numerical reasons. $G_{e}$ and $G_{i}$ are the gyroviscous part of the pressure tensor (see Ref. 31 for their explicit expression). The Poisson brackets are expressed as $[f, g]=\mathbf{b} \cdot(\nabla f \times \nabla g)$, where $\mathbf{b}$ is the unit magnetic field vector and the curvature operator is $\hat{C}(f)=B / 2[\nabla \times(\mathbf{b} / B)] \cdot \nabla f$. In Eqs. (1), and in the remainder of the present paper, we normalize $n$ to the reference density $n_{0}, T_{e}$ to the reference temperature $T_{e 0}, \phi$ to $T_{e 0} / e, V_{\| e}$ and $V_{\| i}$ to $c_{s 0}=\sqrt{T_{e 0} / m_{i}}$ (and therefore $c_{s}$ to $c_{s 0}$ ), and time $t$ to $R / c_{s 0}$. Lengths in the perpendicular direction are adimensionalized to $\rho_{s 0}=c_{s 0} / \Omega_{c i}$ and in the parallel direction to $R$.

For simplicity, we consider the system of Eqs. (1) in $s-$ $\alpha$ circular geometry ${ }^{34}$ with a toroidal limiter positioned on the high field side equatorial midplane of the device. In this geometry, operators are computed in the $\epsilon=a / R \rightarrow 0$ limit ( $a$ is the tokamak minor radius). Therefore, the Poisson brackets reduce to $[f, g]=\partial_{y} f \partial_{r} g-\partial_{r} f \partial_{y} g$, where $r$ is the flux coordinate and corresponds, in a circular magnetic flux surface configuration, to the radial direction, while $y$ is the coordinate perpendicular to $r$ and $\mathbf{B}$. In the $\epsilon \rightarrow 0$ limit, the plane $(r, y)$ coincides with the poloidal plane and, as a consequence, $y=a \theta$, where $0<\theta<2 \pi$ is the poloidal angle, with $\theta=0$ and $\theta=2 \pi$ at the outer midplane. Moreover, the expression of the curvature operator is $\hat{C}(f)=\sin \theta \partial_{r} f+(\hat{s} \theta \sin \theta$ $+\cos \theta) \partial_{y} f$, where $\hat{s}=(a / q) d q / d r$ is the magnetic shear, the perpendicular Laplace operator is $\nabla_{\perp}^{2} f=\partial_{r}^{2} f+2 \hat{s} \theta \partial_{r, y}^{2} f$ $+\left[1+(\hat{s} \theta)^{2}\right] \partial_{y}^{2} f$, and the parallel gradient reads as $\nabla_{\|} f=\partial_{z} f$, where $z$ is the direction parallel to the field lines, $0<z<2 \pi q$. The system of Eqs. (1) is completed by an appropriate set of boundary conditions at the limiter plates, derived in Ref. 32: 


$$
\begin{aligned}
V_{\| i} & = \pm c_{s} \\
V_{\| e} & = \pm c_{s} \exp \left(\Lambda-\phi / T_{e}\right) \\
\frac{\partial n}{\partial y} & =\mp \frac{n}{c_{s}} \frac{\partial V_{\| i}}{\partial y} \\
\frac{\partial \phi}{\partial y} & =\mp c_{s} \frac{\partial V_{\| i}}{\partial y} \\
\frac{\partial T_{e}}{\partial y} & =0 \\
\nabla_{\perp}^{2} \phi & =-\cos ^{2} \alpha\left[\left(\frac{\partial V_{\| i}}{\partial y}\right)^{2}+c_{s} \frac{\partial^{2} V_{\|_{i}}}{\partial y^{2}}\right]
\end{aligned}
$$

where $\Lambda=\log \sqrt{m_{i} /\left(2 \pi m_{e}\right)} \simeq 3, \alpha$ is the angle between the magnetic field and the limiter, and terms related to radial gradients have been neglected.

The system of Eqs. (1) with the boundary conditions in Eqs. (2) is able to describe the quasi-steady state SOL regime which results from the interplay of the plasma outflow from the core, perpendicular transport, and losses at the limiter plates. The estimate of the plasma density scale length in this quasi-steady regime is the focus of Sec. III.

\section{ESTIMATE OF THE SOL PLASMA GRADIENT LENGTH}

In the SOL, the plasma pressure scale length results from a balance between turbulent radial transport and parallel losses. Different possible mechanisms have been proposed to provide saturation of the linear modes during the non-linear phase (see, e.g., Refs. 8, 11, 29, and 35), therefore setting the amplitude of the plasma fluctuations and the related radial turbulence level. In the limit of negligible $\boldsymbol{E} \times \boldsymbol{B}$ shear flow, in Ref. 29, two saturation mechanisms are shown to play a role in the SOL: the growth of the KelvinHelmholtz (secondary) instability and the gradient removal mechanism, i.e., the local flattening of the plasma gradients and associated removal of the instability drive. Analytical estimates and numerical simulations show that the gradient removal saturation mechanism is at play in the typical regime of SOL turbulence. ${ }^{29}$

A complete description of the gradient removal mechanism is given in Ref. 29; here, we summarize its main features. Saturation occurs when the radial gradient of the perturbed density becomes comparable to the radial gradient of the background density, i.e., $k_{r} \tilde{n} \sim \bar{n} / L_{n}$, where $L_{n}$ is the radial length of the background density and $k_{r}$ denotes the typical radial wavevector of the instability. (The tilde indicates fluctuating quantities, while the overbar denotes equilibrium quantities, e.g., $n=\bar{n}+\tilde{n}$.) In the following, we therefore assume $L_{n} \sim L_{p} \sim L_{T}$. The time and poloidal averaged turbulent $\mathbf{E} \times \mathbf{B}$ radial particle flux can be estimated as $\Gamma_{r}=R\left\langle\tilde{n} \partial_{y} \tilde{\phi}\right\rangle_{y} \sim R k_{y} \tilde{\phi} \tilde{n}$, where $k_{y}$ is the poloidal wavenumber of the mode dominating transport. Since the electric potential fluctuation can be evaluated from the leading order terms in the density equation, $\partial_{t} n \simeq-R[\phi, n]$, as $\tilde{\phi} \sim \gamma \tilde{n} L_{n} /\left(\bar{n} R k_{y}\right)$, where $\gamma$ is the linear growth rate of the mode that dominates the turbulent dynamics, we obtain an estimate for the radial flux, $\Gamma_{r} \sim \gamma \bar{n} /\left(k_{r}^{2} L_{n}\right)$. For both DWs and BMs, we can assume $k_{r} \sim \sqrt{k_{y} / L_{n}}$, following non-local linear theory methods outlined in Refs. 24, 36, and 37.

In order to obtain an estimate of $L_{n}$, we write a balance between the radial particle flux and the parallel losses at the limiter plates, i.e., $\partial_{r} \Gamma_{r} \sim \Gamma_{r} / L_{n} \sim \bar{n} c_{s} / q$, as the plasma flux to the limiter can be neglected compared to the parallel one. Substituting the expressions for $\Gamma_{r}$ into the particle balance, we obtain

$$
L_{n} \sim \frac{q}{c_{s}}\left(\frac{\gamma}{k_{y}}\right)_{\max },
$$

where the ratio of the linear growth rate to the poloidal wavenumber has to be maximized over the unstable modes present in the system.

Equation (3) allows us to predict $L_{n}$ as a function of the SOL operational parameters: $m_{e} / m_{i}, \nu, q, R / \rho_{s}$, and $\hat{s}$. For this purpose, we first evaluate the growth rate of the linear modes described by the system of Eqs. (1), as a function of $k_{y}$ and $R / L_{n}$, having fixed the SOL operational parameters. We then maximize $\gamma / k_{y}$ over $k_{y}$, obtaining $\left(\gamma / k_{y}\right)_{\max }$ as a function of $R / L_{n}$ (with all the other parameters fixed). We then seek for the value of $R / L_{n}$ that satisfies Eq. (3), i.e., $\left(\gamma / k_{y}\right)_{\max }=L_{n} c_{s} / q$, obtaining our $L_{n}$ prediction. We note that $\gamma$ is obtained by linearizing Eqs. (1) as

$$
\begin{aligned}
\gamma \tilde{n}= & \frac{R}{L_{n}} i k_{y} \tilde{\phi}+2 \hat{C}\left(\tilde{T}_{e}+\tilde{n}-\tilde{\phi}\right)-\nabla_{\|} \tilde{V_{\| e}}, \\
\gamma \nabla_{\perp}^{2} \tilde{\phi}= & 2 \hat{C}\left(\tilde{n}+\tilde{T}_{e}\right)+\nabla_{\|}\left(\tilde{V_{\| i}}-\tilde{V_{\| e}}\right), \\
\frac{m_{e}}{m_{i}} \gamma \tilde{V_{\| e}}= & \nu\left(\tilde{V_{\| i}}-\tilde{V_{\| e}}\right)+\nabla_{\|}\left(\tilde{\phi}-\tilde{n}-1.71 \tilde{T}_{e}\right), \\
\gamma \tilde{T}_{e}= & \eta \frac{R}{L_{n}} i k_{y} \tilde{\phi}+\frac{4}{3} \hat{C}\left(\frac{7}{2} \tilde{T_{e}}+\tilde{n}-\tilde{\phi}\right) \\
& +\frac{2}{3} 1.71 \nabla_{\|}\left(\tilde{V_{\| i}}-\tilde{V_{\| e}}\right)-\frac{2}{3} \nabla_{\|} V_{\| i}, \\
\gamma \tilde{V}_{\| i}= & -\nabla_{\|}\left(\tilde{n}+\tilde{T}_{e}\right) .
\end{aligned}
$$

In system (4), the gyroviscous part of the stress tensor is neglected; $\eta=L_{n} / L_{T}$ is the ratio of the density to the electron temperature scale length. For simplicity, in the following, we assume $\eta=1$. This is justified by simulation and experimental results showing that $\eta$ is of order unity. In fact, in the non-linear simulation results presented herein, $\eta \simeq 0.7$, which corresponds to the typical value observed in the simulations. ${ }^{37}$ Moreover, limited plasmas realized in the JET, Alcator C-MOD, COMPASS, and Tore Supra tokamaks (Ref. 38 and references therein), covering a wide range of parameter, show $0.3 \lesssim \eta \lesssim 1.25$. We ignore background $\mathbf{E} \times$ B flow ( $\bar{\phi}$ is assumed to be independent of the radial coordinate) and, since $k_{y} / k_{r} \sim \sqrt{k_{y} L_{n}}>1$, we also ignore the radial mode dependence. By writing the perturbed quantities in the form $\tilde{f}=\tilde{f_{k_{y}}}(z) \exp \left(i k_{y} y+\gamma t\right)$, we reduce the system (4) to a one-dimensional eigenvalue problem in the $z$ direction, where the laplacian operator is $\nabla_{\perp}^{2}=-k_{\perp}^{2}=-k_{y}^{2}[1$ $\left.+(z / q \hat{s})^{2}\right]$, and the curvature operator is defined as $\hat{C}=i k_{y} C$, being $C=[\cos (z / q)+\sin (z / q)(z / q) \hat{s}]$. We note that a detailed analysis of the instabilities described by system (4) has been presented in Ref. 28. 

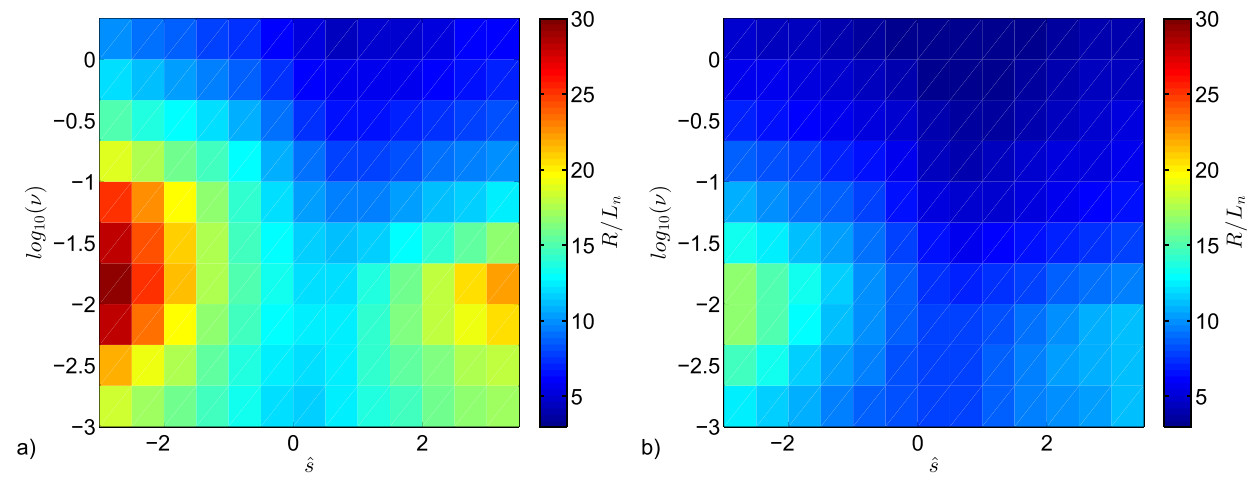

FIG. 1. Gradient removal estimate of $R / L_{n}$, as a function of $\hat{s}$ and $\nu$ for $q=4$ (a), and for $q=8$ (b)
The $R / L_{n}$ estimate as a function of $\nu$ and $\hat{s}$ is showed for $q=4$ in Fig. 1(a) and for $q=8$ in Fig. 1(b) $\left(m_{i} / m_{e}=1836\right)$. We observe that the gradient is steeper for negative $\hat{s}$ and low $\nu$, for both the $q=4$ and the $q=8$ case. Moreover, for $q=4, R / L_{n}$ is higher than in the $q=8$ case.

\section{THE SCRAPE-OFF LAYER TURBULENT REGIMES}

As pointed out by our analysis of the SOL linear modes, ${ }^{28}$ the main instabilities expected to play a role in the SOL are the resistive and inertial branches of the ballooning modes (RBM and IBM) and of the drift waves (RDW and IDW). Ballooning modes have an interchange character and are driven by the presence of magnetic field line curvature and plasma pressure gradients. The dispersion relation that describes the fundamental properties of BMs can be obtained from Eqs. (4) by neglecting coupling with sound waves, plasma compressibility, parallel flows in the density and temperature equations, and the $\nabla_{\|}\left(n+1.71 T_{e}\right)$ term in Ohm's law. The obtained boundary value problem for $\phi$ reads as

$$
-\left[1+\left(\frac{z}{q} \hat{s}\right)^{2}\right] \phi \gamma=2 C \frac{R}{L_{n}} \frac{(1+\eta)}{\gamma} \phi+\frac{1}{\hat{\nu}} \frac{\partial^{2} \phi}{\partial z^{2}},
$$

where $\hat{\nu}=\nu+\gamma m_{e} / m_{i}$. In the limit $\nu \rightarrow 0$, Eq. (5) reduces to the dispersion relation for the IBM, while for $m_{e} / m_{i} \rightarrow 0$ the RBM dispersion relation is retrieved. Both the RBM and IBM growth rates are such that $\gamma / \gamma_{I} \rightarrow 1$, where $\gamma_{I}=\sqrt{2 R / L_{p}}$, respectively, for $\sigma_{R}=1 /\left(\gamma_{I} k_{y}^{2} q^{2} \nu\right) \rightarrow 0$, and $\sigma_{I}=m_{i} /\left(\gamma_{I} k_{y} q \sqrt{m_{e}}\right) \rightarrow 0 .^{28} \quad$ Stabilization of BMs is observed for $k_{y} \gtrsim 0.3 \gamma_{I}$, due to plasma compressibility. The effect of magnetic shear is a reduction of the growth rate for $\hat{s} \lesssim 0$ and $\hat{s} \gtrsim 1$. $^{28}$
The DW instability is driven by the $\mathbf{E} \times \mathbf{B}$ convection of the background pressure gradient, coupled with the breaking of the electron adiabaticity due to finite resistivity or finite electron mass. In order to describe the fundamental properties of the DW, the following equation for $\phi$ can be used:

$$
\gamma k_{\perp}^{2} \phi=\frac{1}{\hat{\nu}} \frac{\partial^{2} \phi}{\partial z^{2}}+\frac{2.94}{\hat{\nu}} \frac{\partial^{2}\left(k_{\perp}^{2} \phi\right)}{\partial z^{2}}-\frac{1}{\hat{\nu} \gamma}\left[i k_{y}(1+1.71 \eta)\right] \frac{\partial^{2} \phi}{\partial z^{2}},
$$

where the curvature terms in Eqs. (4) are neglected as well as the coupling with sound waves. In Eq. (6), we retrieve the dispersion relation for the IDW, in the limit $\nu \rightarrow 0$, and the RDW dispersion relation for $m_{e} / m_{i} \rightarrow 0$. Typically, $\gamma \sim \omega_{*}$, where $\omega_{*}=k_{y} R / L_{n}$ is the diamagnetic frequency, $k_{y} \sim 1$ and $k_{\|}$assumes a finite value.

Which of these instabilities drives the SOL turbulent dynamics? The goal of the present paper is to describe the nonlinear turbulent regimes as a function of the SOL operational parameters, i.e., to understand the nature of the instability responsible for the largest fraction of the radial transport. This is achieved by evaluating the growth rate of the IBM, RBM, IDW, and RDW, the inertial and resistive limits of Eqs. (5) and (6), as a function of the SOL operational parameters, at the $k_{y}$ and $R / L_{n}$ given by Eq. (3). The turbulent regime is defined according to the instability among those four that has the highest $\gamma / k_{y}$ value.

In Fig. 2, different colors are used to represent the nonlinear turbulent regimes at $q=4$ and $q=8$. At both values of $q$, we retrieve some of the linear results of Ref. 28: DWs are the dominant instability at low $\nu$ and negative $\hat{s}$, where $R / L_{n}$ is high. At $\nu \lesssim 10^{-2}$, the dominant instability is the IDW. On the other hand, the BM regime extends in the region where
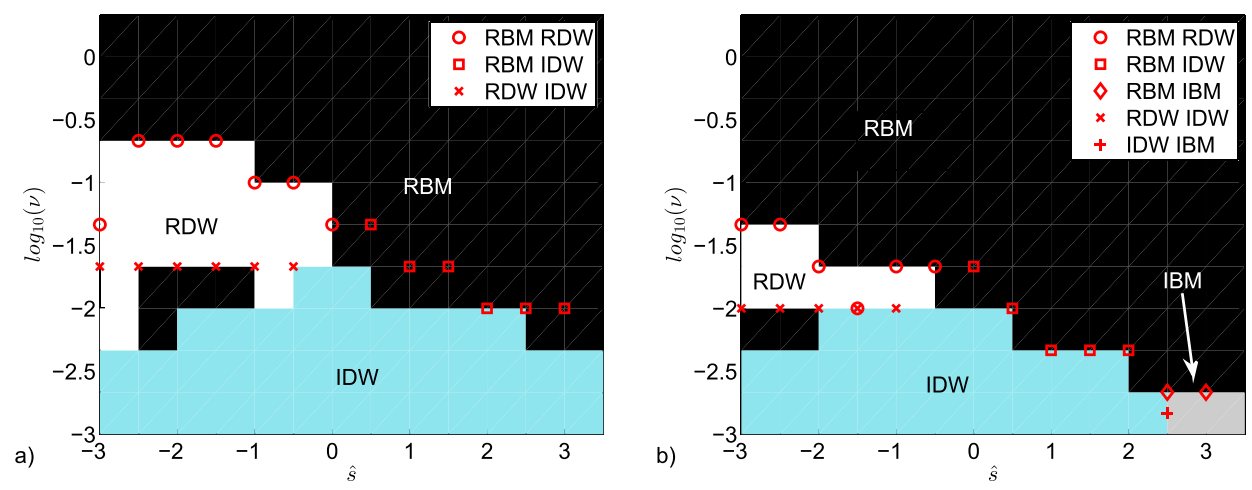

FIG. 2. Turbulent regimes for $q=4$ (a), and for $q=8$ (b); different colors identify different regimes: RBM (black), IBM (grey), IDW (light blue), and RDW (white). The red symbols indicate the estimate of the transition between regimes obtained in Sec. IV. 
gradients are more relaxed $(\hat{s}>0$ and large $\nu)$. We also remark that, with respect to $q=4$, the safety factor $q=8$ favours BMs. This is in agreement with Ref. 28, which shows that the $R / L_{n}$ value at which the transition from BMs to DWs occurs is a decreasing function of $\sigma_{R}$ and $\sigma_{I}$, meaning that, at higher $q$, steeper gradients are needed to develop DWs. We finally note that, at $q=8$, the IBM dominates at the lowest values of $\nu$ and positive $\hat{s}$, while it is not present at $q=4$.

In order to provide a complete and general estimate of the parameter ranges where the different instabilities dominate, we proceed to a more detailed analysis of the transition between the instabilities. More precisely, we evaluate the location in the operational parameters space of the five transitions observed in Fig. 2: RBM and IBM, RBM and IDW, RBM and RDW, RDW and IDW, and IDW and IBM.

We first consider the transition between the RBM and the IBM. We use the dispersion relations of the RBM and IBM [the resistive and inertial limits of Eq. (5)] to obtain, separately for these two branches, the expected $R / L_{n}$ and the $\gamma / k_{y}$ of the mode dominating the non-linear dynamics. The transition between the RBM and IBM regimes takes place when their $\gamma / k_{y}$ are equal, at a $\nu$ value that depends on $\hat{s}$ and $q$, which is plotted in Fig. 3(a). We note that the white region in Fig. 3(a) represents the parameter region in which the RBM dominates over the IBM independently of $\nu$. This region extends at $\hat{s}<0$. BMs are, in fact, suppressed by negative shear, and the stabilisation is more efficient for the IBM than for the RBM. For $\hat{s}>0$, as the threshold occurs at $\nu \sim 1 \times 10^{-3}$, we expect the RBM to prevail over the IBM in typical experimental conditions, where $\nu \sim 10^{-2}-10^{-3}$

Following a similar procedure and considering the resistive and inertial limits of Eqs. (5) and (6), respectively, it is possible to evaluate the transition between the RBM and the
IDW. This is shown in Fig. 3(b), which provides the value of $\nu$ above which the RBM prevails over the IDW. We observe that the RBM dominates at positive values of $\hat{s}$ and high $q$, which are favourable to its growth, as previously noted.

In Fig. 3(c), we also show the value of $\nu$ above which the RBM prevails over the RDW. This is evaluated considering the resistive limit of Eqs. (5) and (6). The $\nu$ threshold diminishes with increasing $q$ and $\hat{s}$. In Ref. 28 , it is noticed that the RBM dominates over the RDW for highly positive and highly negative values of $\hat{s}$, and for high values of $q$, which corresponds to low $\sigma_{R}$. These predictions agree with the findings showed in Fig. 3(c). In the white region, the RBM prevails on the RDW for all values of $\nu$.

In order to accurately describe the transition between RDW and IDW, we follow a slightly different procedure. In fact, as the growth rate of IDW is sensitive to $k_{y}$, and $k_{y}$ can be affected by the inclusion of even a small resistivity, we cannot decouple the two instabilities. Therefore, we compute the expected $R / L_{n}$ by considering, for the gradient removal mechanism, the linear growth rate given by Eq. (6), which includes both RDW and IDW. At the $R / L_{n}$ and $k_{y}$ found, we then calculate the RDW and IDW growth rates, which we compare, finding the value of $\nu$ above which the RDW prevail over the IDW, as a function of $\hat{s}$ and $q$. This is shown in Fig. 3(d). We note that the transition is symmetric with respect to $\hat{s}=0$, as a consequence of the symmetry of Eq. (6). The RDW onsets at values of $\nu$ decreasing with $q$. This is different than the conclusions reported in Ref. 28, where the transition between the peak growth rate of two instabilities was shown to depend on $\hat{s}$ and to be independent of $q$.

Finally, we consider the transition between the IBM and the IDW. To estimate this transition, we consider the inertial limits of Eqs. (5) and (6), which are independent of $\nu$, as it is our estimate of the transition between these two instabilities.
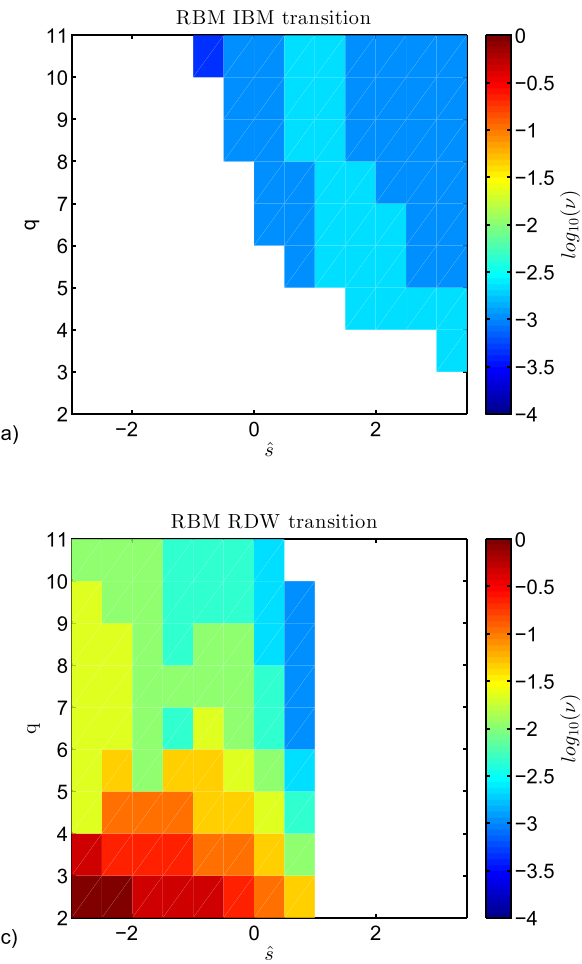

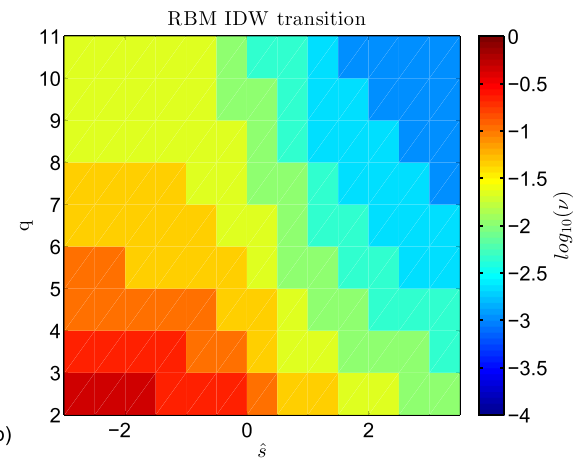

RDW IDW transition

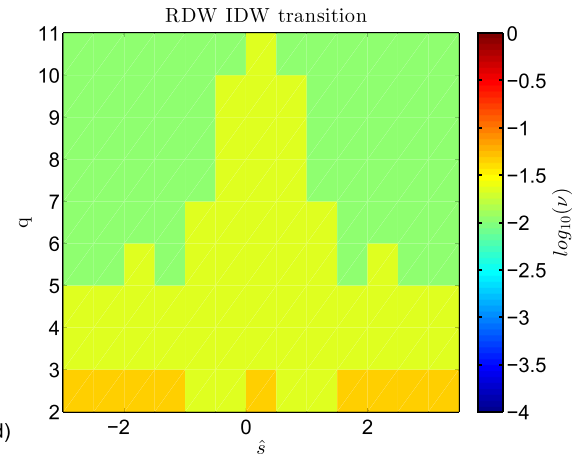

FIG. 3. Value of $\nu$, as a function of $\hat{s}$ and $q$, of the transition between RBM and IBM (in the white region RBM prevails on IBM independently of $\nu$ ) (a), transition between RBM and IDW (b), transition between RBM and RDW (in the white region RBM prevails on RDW independently of $\nu$ ) (c), and transition between RDW and IDW (d). In all cases, the first instability prevails over the second one at $\nu$ values larger than the ones plotted. 
We find that, for $\hat{s} \lesssim 1$, the IDW prevail over the IBM for all the values of $q$. For $\hat{s} \gtrsim 1$, IBM dominates over IDW above a $q$ values that varies approximately linearly from $q \simeq 10$ at $\hat{s}=1$ to $q \simeq 7$ at $\hat{s}=3$. The IBM is therefore the leading instability for high $q$ and for $\hat{s}>0$; this generally agrees with the observations presented in Ref. 28.

We can now use the transition estimates discussed above to explain the SOL non-linear regimes displayed in Fig. 2. Our estimates are plotted by using red symbols, showing a good agreement with the observed transitions. For $q=4$ and for $q=8$, for $\hat{s}<0$, from low to high values of $\nu$, we essentially observe the transition between three regimes: IDW, RDW, and RBM. According to the results in Figs. 3(d) and $3(\mathrm{c})$, the transition between the IDW and the RDW, and between the RDW and the RBM, respectively, occur at higher $\nu$ for $q=4$ with respect to $q=8$.

For $\hat{s}>0$, we observe that the RDW regime disappears as the RBM prevails on the RDW [see Fig. 3(c)]. At $q=4$, we observe the presence of two regimes; IDW and RBM from low to high $\nu$, while at $q=8$, at the highest $\hat{s}$ and lowest $\nu$ values, also the IBM instability appears, in agreement with the results in Fig. 3(a). We also observe that the RBM prevails on the IDW for smaller values of $\nu$ with respect to the $q=4$ case [see Fig. 3(b), where the $\nu$ threshold between the RBM and the IDW decreases with increasing $q$ ]. Finally, we note that the application of our SOL turbulent regime analysis for predicting the turbulent regime of a typical L-mode discharge in the TCV tokamak ${ }^{39}$ (for $\nu \simeq 10^{-2}, q \simeq 5$ and $\hat{s} \simeq 2$ ) points to the RBM regime.

\section{NON-LINEAR SIMULATIONS}

In Secs. III and IV, the equilibrium gradient and the instability regimes are predicted, based on the gradient removal theory and the evaluation of the linear growth rate. Here, we present the results of non-linear simulations of SOL turbulence that support our methodology to determine the SOL turbulent regimes.

The simulations have been performed using the GBS code, described in Ref. 31. The code was initially conceived for simulating plasma turbulence in basic plasma physics devices (see, e.g., Refs. 24, 25, 37, 40-42), and it has been validated with the experimental results from the TORPEX device. ${ }^{41,43}$ It has been further developed in order to describe SOL turbulence. ${ }^{29-31}$ Since in the SOL fluctuations are comparable to background quantities, the code solves Eqs. (1), with boundary conditions given by Eqs. (2), without separation of background and fluctuation quantities. Therefore, the background pressure gradient is not fixed a priori and it results from the self-consistent evolution of the plasma profiles.

Typical SOL simulations results are described in Ref. 31 . The plasma outflow from the core is mimicked by a density and a temperature source, $S_{n}$ and $S_{T}$, defined as $S_{n, T}=\exp \left\{-\left[\left(r-r_{s}\right)^{2} / \sigma_{s}^{2}\right]\right\}$, with $r_{s}=30$ and $\sigma_{s}=2.5$. Other simulation parameters are the major radius, $R=500$, and the domain dimensions, $L_{r}=100$ and $L_{y}=800$. After an initial transient, a non-linear quasi-steady regime is reached, as a balance between plasma outflow from the core, turbulent transport and parallel losses at the vessel. Our analysis is focused on this quasi-steady state regime. Among a number of simulations that we have carried out, we focus and we present the results of four simulations that belong to the four predicted instability regimes: RBM, IBM, RDW, and IDW. The plasma parameters of these four simulations are listed in Table I. We first estimate the equilibrium $R / L_{n}$, using the gradient removal theory, and we compare our prediction with the results of the non-linear simulations. As reported in Table I, our estimates show reasonable agreement with the simulations results, the maximum relative error ranging from $10 \%$ to $25 \%$. In Table I, we also compare the gradient-removal predicted $k_{y}$ of the dominant mode with the time averaged $k_{y}$ of the mode leading to the maximum turbulent flux in the simulations. The uncertainty affecting $k_{y}$ is estimated by considering a $\pm 10 \%$ variation of the $\gamma / k_{y}$ value with respect to its maximum at the predicted $R / L_{n}$ (for comparison, we note that the standard deviation of the time averaged particle flux, proportional to $\gamma / k_{y}$, is approximately $25 \%$ of the time averaged particle flux). We verify that all the non-linear simulations studied herein satisfy the inequality $\sqrt{k_{y} L_{n}}>1$, and that they belong

TABLE I. Overview of the non-linear simulations input parameters and results. The radial window over which the non-linear $R / L_{n}$ and $k_{y}$ are evaluated is $5<r-r_{s}<17$. The two values $k_{y, \min }$ and $k_{y, \max }$ are computed considering the $k_{y}$ range corresponding to a $\pm 10 \%$ variation of the value $\gamma / k_{y}$ with respect to its maximum at the $R / L_{n}$ and $k_{y}$ predicted.

\begin{tabular}{|c|c|c|c|c|c|c|c|c|c|c|c|c|c|c|c|}
\hline Simulation & $\nu$ & $m_{e} / m_{i}$ & $\hat{s}$ & $q$ & ID & $\begin{array}{c}R / L_{n} \\
\text { simulation }\end{array}$ & $\begin{array}{l}\quad R / L_{n} \\
\text { estimated }\end{array}$ & $\begin{array}{c}k_{y} \\
\text { simulation }\end{array}$ & $\begin{array}{c}k_{y} \\
\text { estimated }\end{array}$ & $\begin{array}{c}\quad k_{y, \min } \\
\text { estimated }\end{array}$ & $\begin{array}{c}k_{y, \max } \\
\text { estimated }\end{array}$ & $\gamma_{R B M}$ & $\gamma_{I B M}$ & $\gamma_{R D W}$ & $\gamma_{I D W}$ \\
\hline RBM & 0.5 & $1 / 800$ & 1 & 8 & on & 4.41 & 3.71 & 0.09 & 0.05 & 0.03 & 0.07 & 2.28 & $\simeq 0$ & 0.12 & $\simeq 0$ \\
\hline $\mathrm{RBM}$ reduced $m_{e} / m_{i}$ & 0.5 & $1 / 1600$ & 1 & 8 & on & 4.30 & $\ldots$ & $\ldots$ & $\ldots$ & $\ldots$ & $\ldots$ & $\ldots$ & $\ldots$ & $\cdots$ & $\ldots$ \\
\hline RBM without ID & 0.5 & $1 / 800$ & 1 & 8 & off & 12.12 & $\ldots$ & $\ldots$ & $\ldots$ & $\ldots$ & $\ldots$ & $\ldots$ & $\ldots$ & $\ldots$ & $\ldots$ \\
\hline IBM & 0.005 & $1 / 50$ & 1 & 8 & on & 5.23 & 5.74 & 0.09 & 0.14 & 0.10 & 0.19 & 0.64 & 2.88 & 0.01 & 1.05 \\
\hline $\mathrm{IBM}$ reduced $\nu$ & 0.0005 & $1 / 50$ & 1 & 8 & on & 5.64 & $\ldots$ & $\ldots$ & $\ldots$ & $\ldots$ & $\ldots$ & $\ldots$ & $\ldots$ & $\ldots$ & $\ldots$ \\
\hline IBM without ID & 0.005 & $1 / 50$ & 1 & 8 & off & 14.57 & $\ldots$ & $\ldots$ & $\ldots$ & $\ldots$ & $\ldots$ & $\ldots$ & $\ldots$ & $\ldots$ & $\ldots$ \\
\hline RDW & 0.05 & $1 / 800$ & -0.7 & 4 & on & 16.30 & 12.46 & 0.13 & 0.18 & 0.14 & 0.24 & 1.20 & $\simeq 0$ & 1.46 & $\simeq 0$ \\
\hline $\mathrm{RDW}$ reduced $m_{e} / m_{i}$ & 0.05 & $1 / 1600$ & -0.7 & 4 & on & 17.01 & $\ldots$ & $\ldots$ & $\ldots$ & $\ldots$ & $\ldots$ & $\ldots$ & $\ldots$ & $\ldots$ & $\ldots$ \\
\hline RDW without ID & 0.05 & $1 / 800$ & -0.7 & 4 & off & 18.39 & $\ldots$ & $\ldots$ & $\ldots$ & $\ldots$ & $\ldots$ & $\ldots$ & $\ldots$ & $\ldots$ & $\ldots$ \\
\hline IDW & 0.005 & $1 / 200$ & -1 & 4 & on & 16.99 & 12.49 & 0.18 & 0.2 & 0.17 & 0.25 & 0.13 & $\simeq 0$ & 0.03 & 2.92 \\
\hline IDW reduced $\nu$ & 0.0005 & $1 / 200$ & -1 & 4 & on & 16.68 & $\ldots$ & $\ldots$ & $\ldots$ & $\ldots$ & $\ldots$ & $\ldots$ & $\ldots$ & $\ldots$ & $\ldots$ \\
\hline IDW without ID & 0.005 & $1 / 200$ & -1 & 4 & off & 13.81 & $\ldots$ & $\ldots$ & $\ldots$ & $\ldots$ & $\ldots$ & $\ldots$ & $\ldots$ & $\ldots$ & $\ldots$ \\
\hline
\end{tabular}


to the regime where the gradient removal mechanism is responsible for turbulence saturation. In Table I, we list the growth rate of each instability separately, in order to identify the regime of the four simulations. A detailed description and analysis of the properties of the non-linear simulations follows, in order to identify and discuss the nature of the transport.

Figs. 4-7 show the density equilibrium profiles, $\bar{n}$, and typical snapshots of the density fluctuation, $\tilde{n}$, in the $(r, y)$ plane, for the identified RBM, IBM, RDW, and IDW simulations, respectively. We define $\bar{n}$ as the time and toroidal average of the density, evaluated during the quasi-steady state phase of the simulation, and we calculate the fluctuating part of the density as $\tilde{n}=n-\bar{n}$. We now discuss a number of tests that show that it is justified to identify the turbulent regime according to the procedure used in Sec. IV.

First, for an identified resistive mode, a simulation with a reduced value of $m_{e} / m_{i}$ is performed, or, for an identified
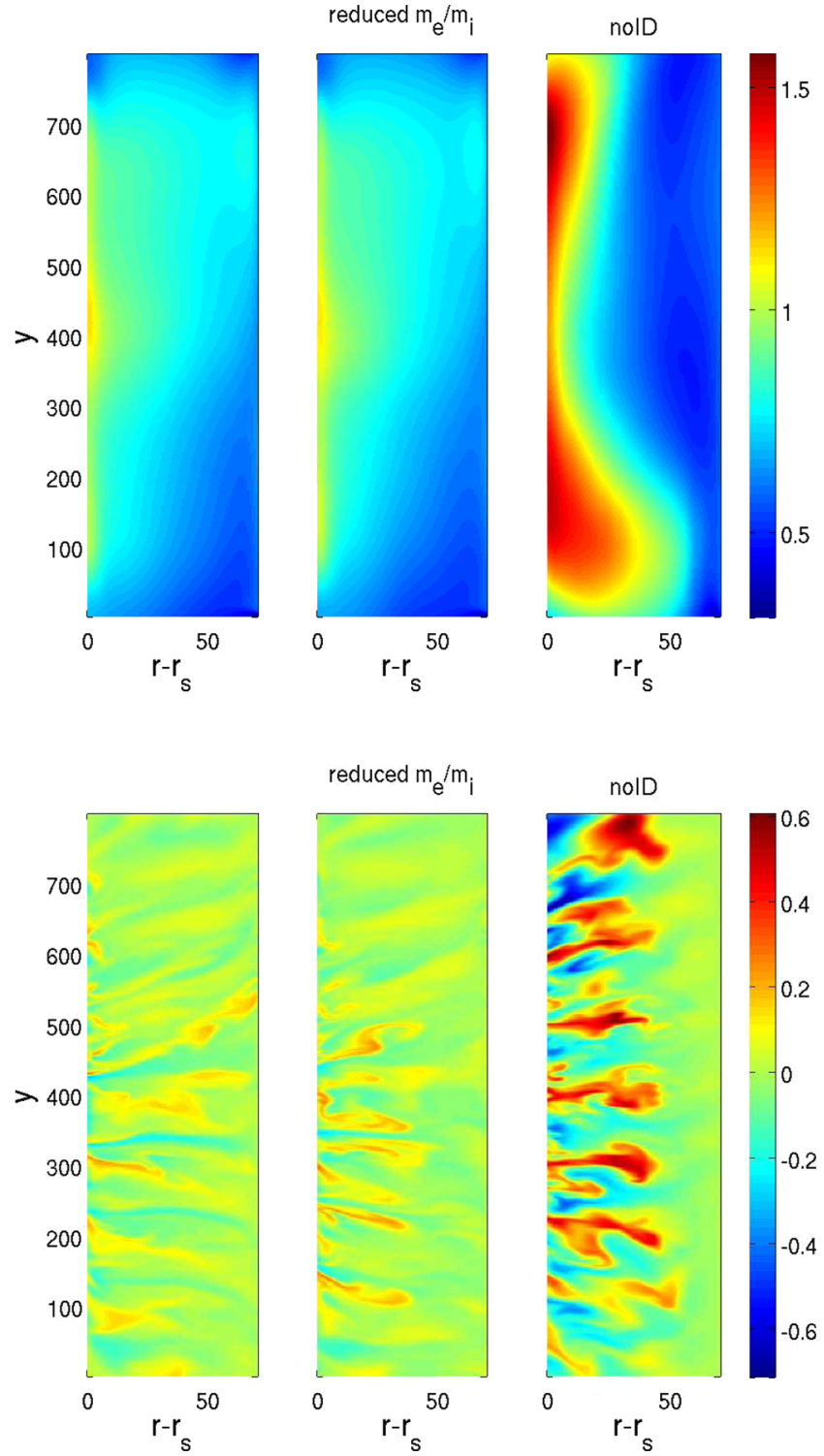

FIG. 4. Density equilibrium profile (top panels) and density fluctuation profile (bottom panels) in the $r-y$ plane for RBM simulation (left), the RBM with reduced $m_{e} / m_{i}$ (center), and the RBM without ID (right). $r_{s}$ radial position of the last closed flux surface, where the plasma source is located. inertial mode, we carry out a simulation with reduced $\nu$. As shown in Figs. 4-7, the change in the radial equilibrium gradient length and the radial extent of the fluctuations is small, confirming our prediction of being in a resistive or inertial regime.

Second, in the four simulations considered, we turn off the interchange drive (ID), i.e., the curvature terms in the vorticity equation. We can infer the BM nature of turbulence, by observing major effects following the ID turn off, while small changes point to a DW regime. For BM simulations, we remark that the average profiles in Figs. 4 and 5 lose their ballooning character once the ID is turned off, $R / L_{n}$ becomes steeper, and the long streamers are broken into smaller structures in the case without ID (therefore, $k_{r}$ increases). This is due to the fact that, while $k_{y}$ does not change significantly, $L_{n}$ decreases from the base case to the case without ID, and therefore $k_{r}$ increases, according to the non-local estimate of the radial eddy extension. For DW simulations, instead, there is no observable difference between the equilibrium profiles, following the ID turn off, and the nature of the fluctuations is
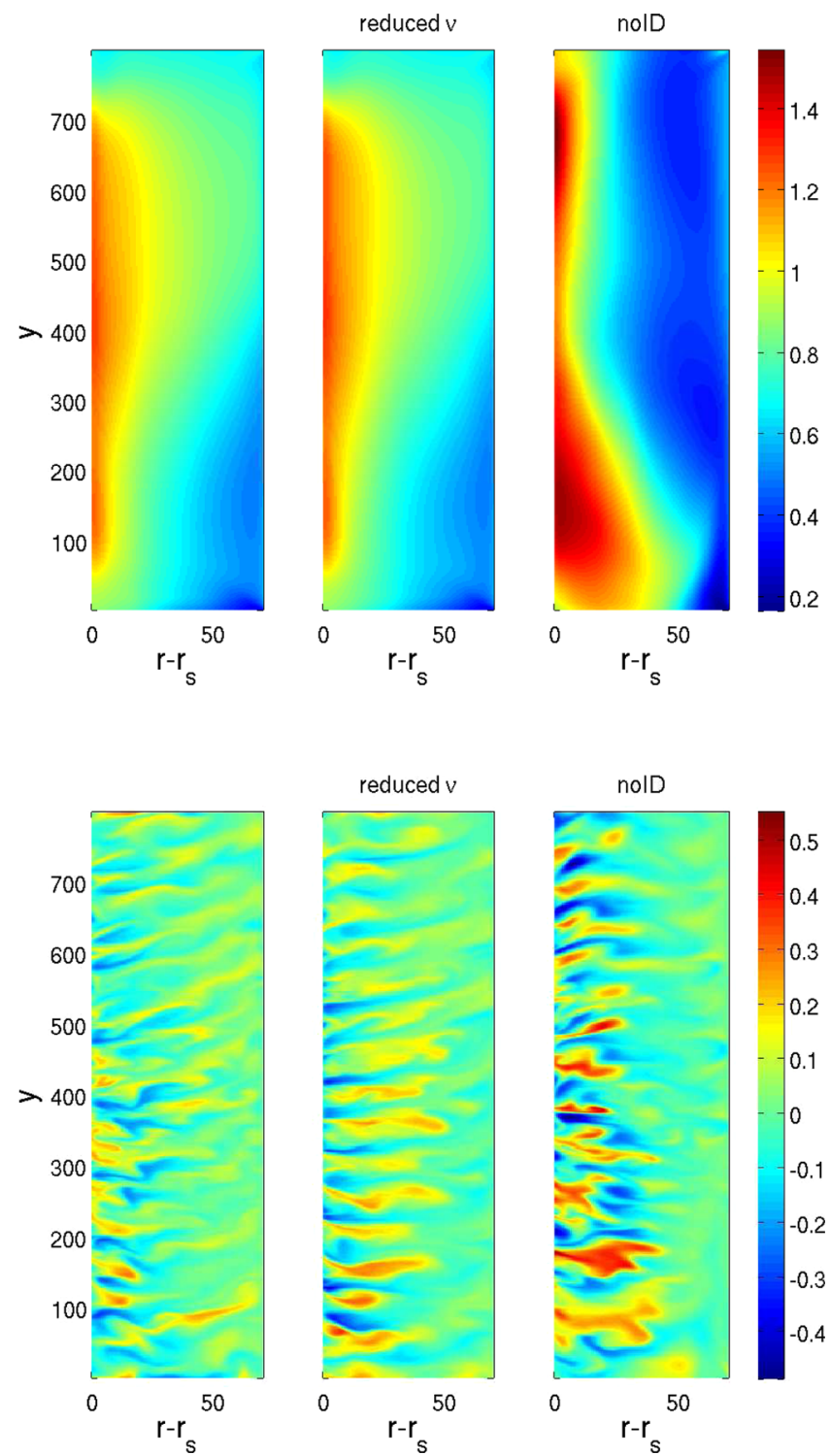

FIG. 5. Same as Fig. 4, for the IBM. 


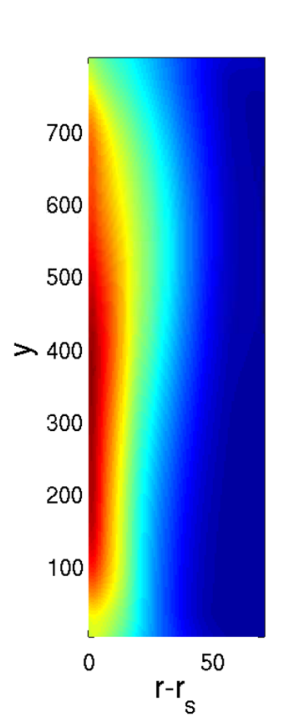

reduced $m_{e} / m_{i}$
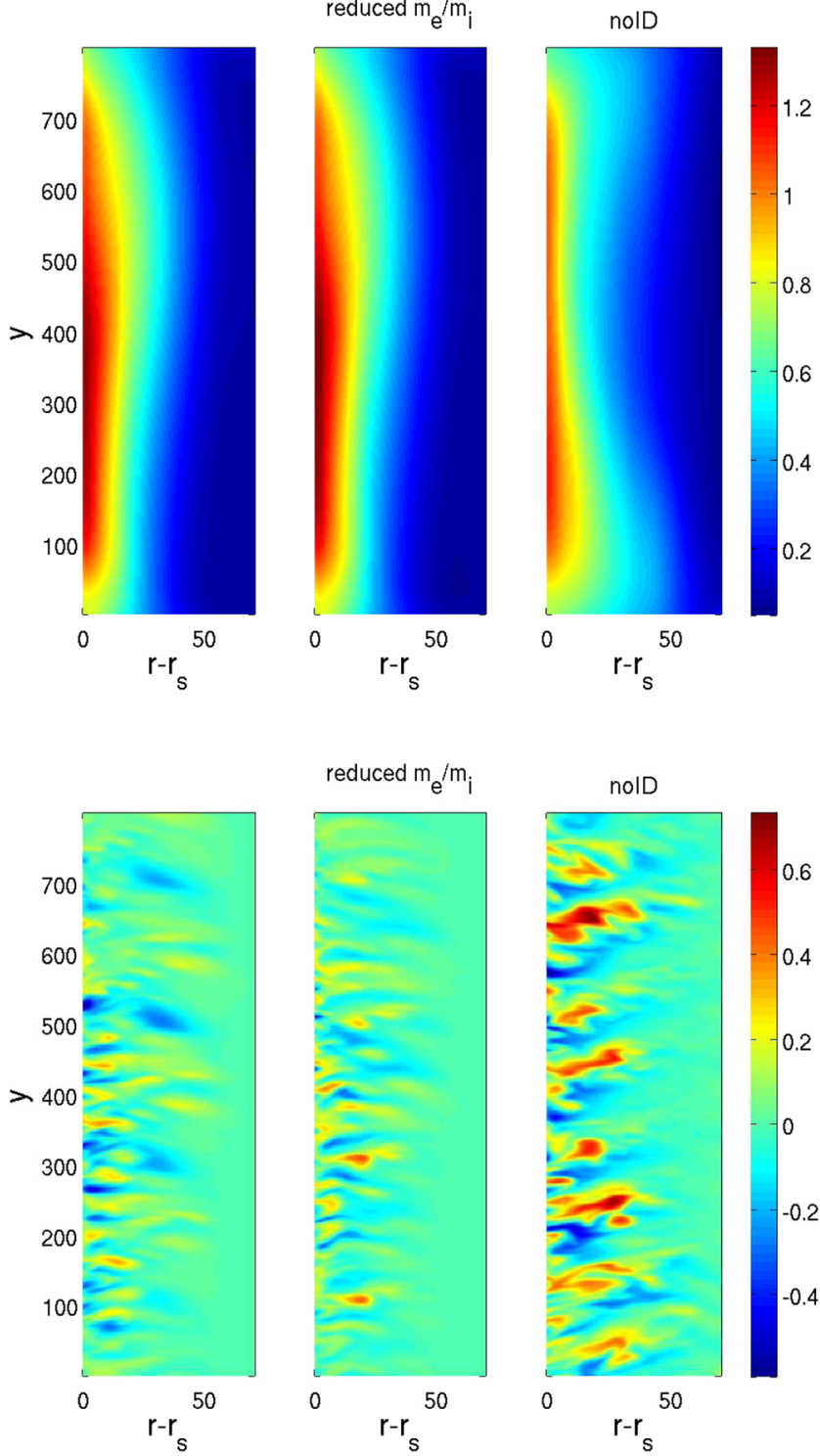

FIG. 6. Same as Fig. 4, for the RDW.

very similar (see Figs. 6 and 7). Moreover, in the simulation with and without ID, the plasma profile is weakly dependent on the poloidal angle, showing a non-ballooning character. We conclude from this analysis that our simulations can be classified as BM or DW dominated, as pointed out by our methodology.

Finally, in order to reinforce the validity of our analysis, we analyze the relation between potential and density fluctuations, according to the methods proposed in Refs. 3 and 8 . For BMs, the vorticity equation imposes a $\pi / 2$ phase shift between $\phi$ and $n$ fluctuations, which are not correlated. In case of DWs, the electrons are close to adiabaticity and the amplitudes of $\phi$ and $n$ fluctuations are clearly correlated. ${ }^{8,9}$ Following Ref. 8, we introduce two analysis techniques to investigate the relation between $\phi$ and $n$ : the phase shift probability and the cross coherence analysis.

The phase shift probability is calculated at a fixed radial position, by considering the FFT along $y$ of the $\phi$ and $n$ fluctuations, as a function of toroidal position and time. From the FFT, we then compute the phase shift, $-\pi \leq \chi<\pi$,
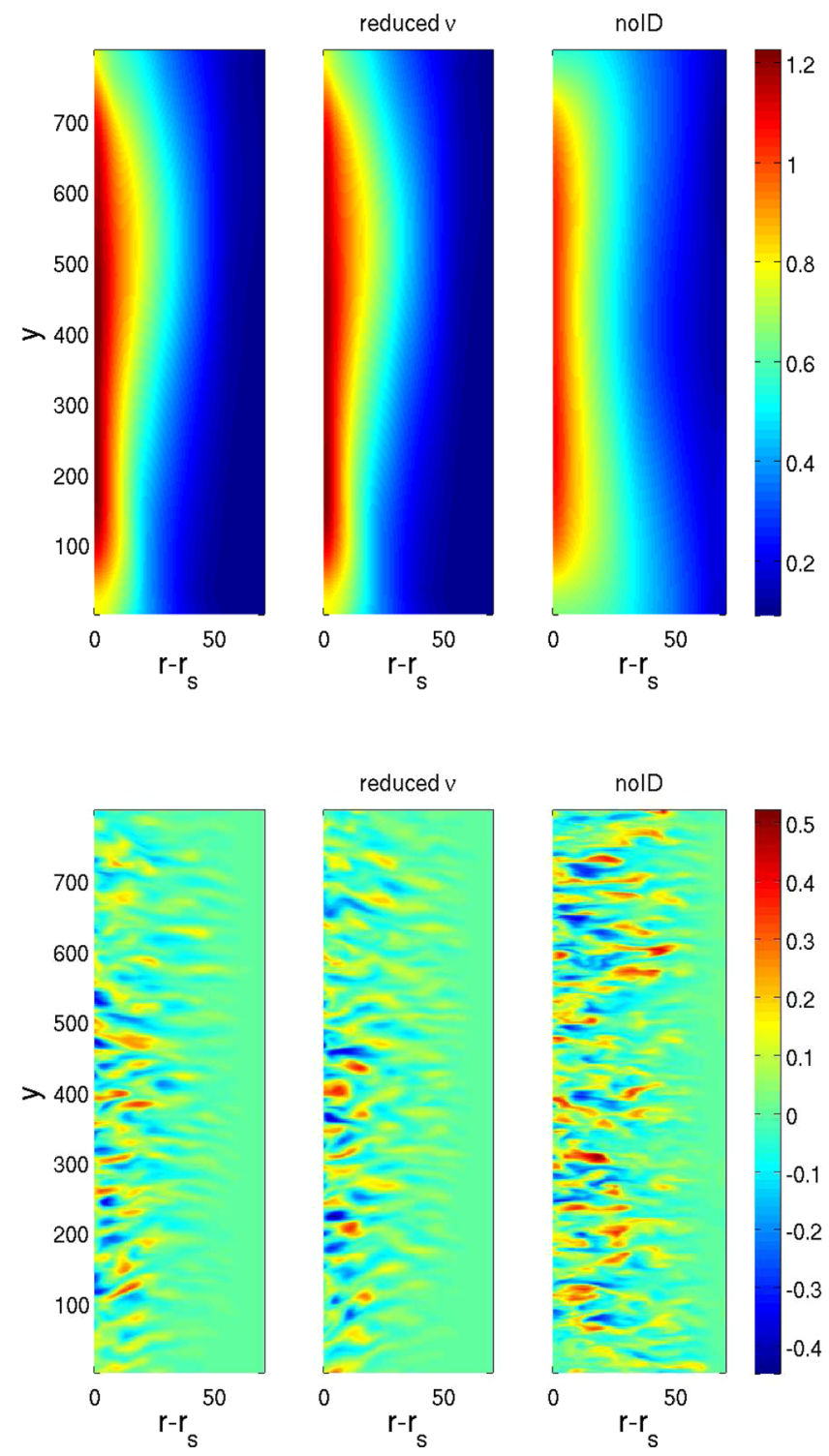

FIG. 7. Same as Fig. 4, for the IDW

corresponding to each $k_{y}$, and we bin them as a function of the toroidal position and time, with the proper weight given by the power spectral density of the $\phi$ and $n$ fluctuations. The phase shift probability between $\phi$ and $n$ is showed in Fig. 8. As expected, for the BM simulations [Figs. 8(a) and 8(b)], the phase shift has a maximum at $\chi \approx 0.5 \pi$ for the dominant mode $k_{y} \approx 0.1$. For DW simulations [Figs. $8(\mathrm{c})$ and 8(d)], we observe a phase shift with a maximum at $\chi \approx 0$ for the dominant mode $k_{y} \approx 0.1$.

The cross coherence is computed at a fixed radial position. The $\phi$ and $n$ fluctuations are considered as a function of the poloidal and toroidal directions, and time, and normalized to their standard deviation. We then evaluate the probability of finding both fluctuations at a certain ordered pair of amplitudes and we display it in Fig. 9. The cross coherence in Fig. 9(a), for the RBM, and Fig. 9(b), for the IBM, does not show correlation between $\phi$ and $n$, while the cross coherence in Fig. 9(c), for the RDW, and Fig. 9(d), for the IDW, shows a high correlation between $\phi$ and $n$ fluctuations. This additional analysis supports our methodology to identify the turbulent regime of the non-linear simulations. 

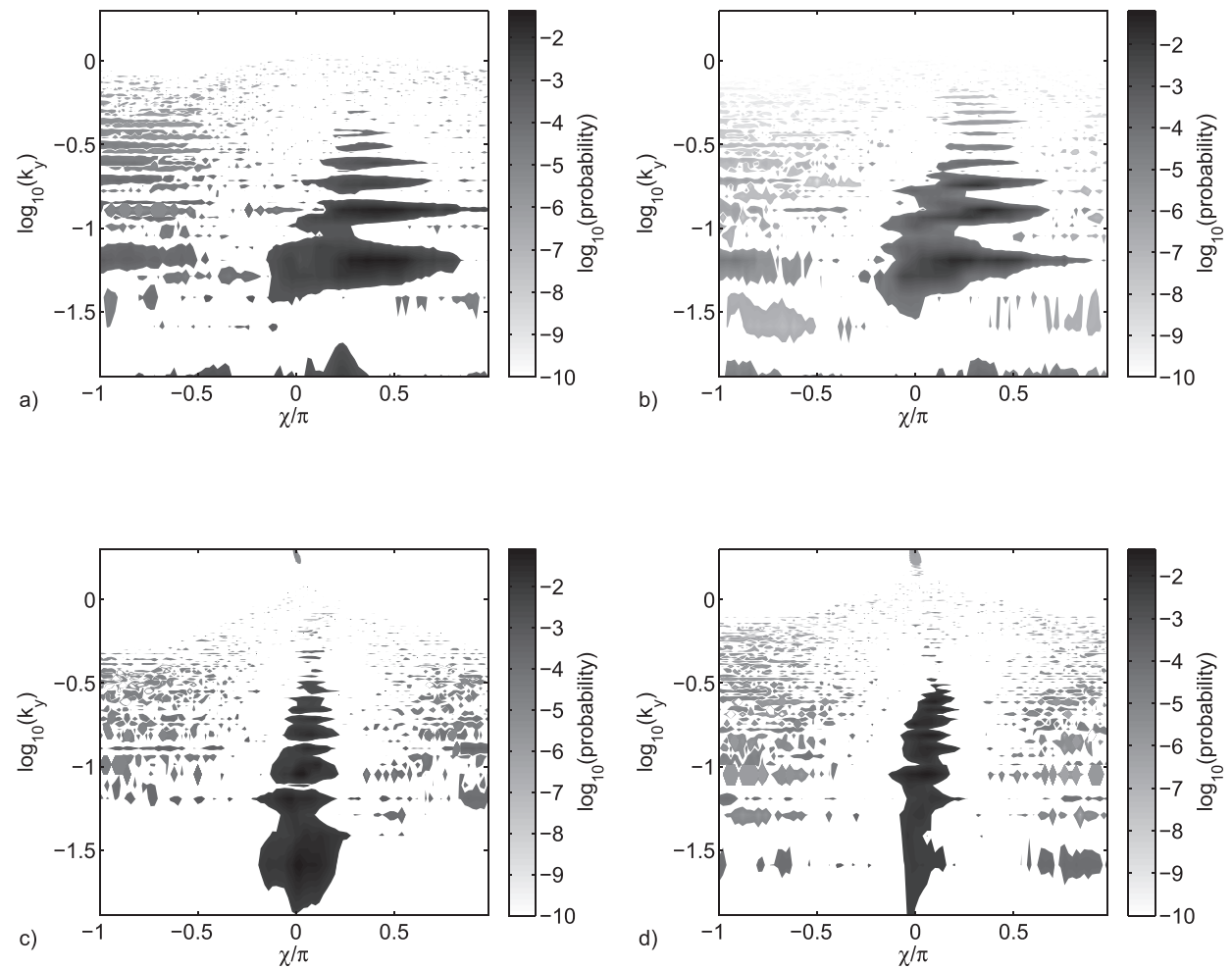

FIG. 8. Phase shift probability between $\tilde{\phi}$ and $\tilde{n}$ weighted according to the power spectral density for the RBM (a), IBM (b), RDW (c), and IDW (d).

\section{CONCLUSIONS}

In the present paper, we have identified the non-linear SOL turbulent regimes as a function of the SOL operational parameters $\left(q, \nu, \hat{s}\right.$, and $\left.m_{i} / m_{e}\right)$ depending on the instability responsible for the non-linear transport. The SOL plasma dynamics has been described by the electrostatic drift-reduced Braginskii equations with cold ions, in the infinite aspect ratio limit with a toroidal limiter at the equatorial high-field side midplane. We have assumed that the linear instabilities are saturated when the plasma pressure gradient is nonlinearly flattened by the growth of the unstable modes. This has allowed us to predict the time-averaged plasma gradient length, which is proportional to $\gamma / k_{y}$, where $\gamma$ is the linear growth rate and $k_{y}$ the poloidal wavenumber of the instability that dominates the non-linear dynamics.

We note that a number of modes are possibly unstable in the edge and SOL regions of tokamak plasmas. While the
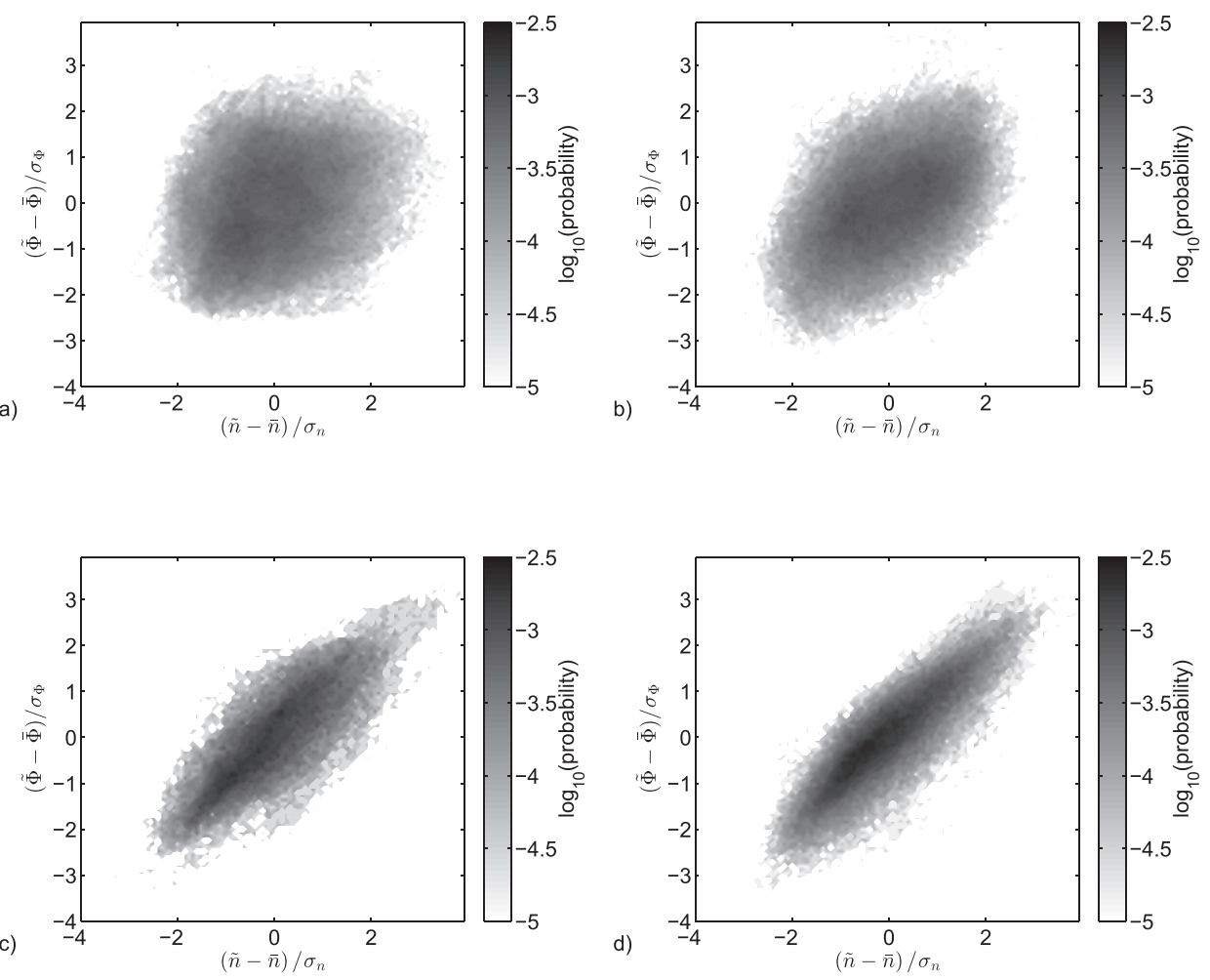

FIG. 9. Cross coherence between $\tilde{\phi}$ and $\tilde{n}$ for the RBM (a), IBM (b), RDW (c), and IDW (d). 
instabilities playing a major role in the tokamak SOL are believed to be the resistive and inertial branches of BMs and DWs, peeling-ballooning modes, external kinks, and sheath modes ${ }^{4-6}$ might also become unstable. In the cold-ion regime considered here, ion temperature gradient $\operatorname{modes}^{2,22}$ are excluded, while trapped electron modes are also stable in the SOL due to the fact that the bounce frequency of trapped electrons is smaller than the collision frequency.

In the present study, we have focused our attention exclusively on the resistive and inertial branches of BMs and DWs. Using simplified models that retain the basic linear characteristics of these instabilities, we have built a map in the operational parameter space, defining the region in which each instability drives transport [see Figs. 2(a) and 2(b) for $q=4$ and $q=8$ ]. We have observed that DWs prevail at negative shear, IDW dominates at low $\nu$, while positive shear and high $q$ are favourable for BMs. We have investigated the transition among the different instabilities (the RBM-IBM, the RBM-IDW, the RBM-RDW, and the RDW-IDW transitions) determining, in general, the threshold value of $\nu$ at which they take place. This is shown in Fig. 3. Being the transition between the IBM and the IDW independent of $\nu$, we have estimated the value of $q$ at which this transition takes place as a function of $\hat{s}$. The estimates are in good agreement with the transitions observed with the full model.

In order to verify the validity of our methodology, we have performed a set of non-linear simulations, and we have presented four of those, each belonging to a different instability regime. The simulations have been carried out with GBS, a global, non-linear code that solves the drift-reduced Braginskii equations. For each set of SOL parameters of the non-linear simulations, we have predicted the instability regime, $R / L_{n}$, and the $k_{y}$ of the saturated non-linear mode, according to the gradient removal hypothesis. The predictions and the results of the non-linear simulations show reasonable agreement. In particular, the analysis of the turbulence character (see Figs. 4-9) supports our methodology to identify the non-linear turbulent regimes.

We remark that our analysis leads not only to the identification of the SOL turbulence regimes but also to the prediction of the steady state gradient and poloidal wavelength at saturation, and therefore to the prediction of the main turbulence properties. The model that we have presented is relatively simple and constitutes a framework which can be generalized to the analysis of more complicated SOL configurations.

\section{ACKNOWLEDGMENTS}

Part of the simulations presented herein were carried out at the Swiss National Supercomputing Centre (CSCS) under project ID s346; and part were carried out using the HELIOS supercomputer system at Computational Simulation Centre of International Fusion Energy Research Centre (IFERC-CSC), Aomori, Japan, under the Broader Approach collaboration between Euratom and Japan, implemented by Fusion for Energy and JAEA. This research was supported by the Swiss National Science Foundation.
${ }^{1}$ A. Loarte, B. Lipschultz, A. Kukushkin, G. Matthews, P. Stangeby, N. Asakura, G. Counsell, G. Federici, A. Kallenbach, K. Krieger et al., Nucl. Fusion 47, S203 (2007).

${ }^{2}$ A. Zeiler, "3D fluid simulations of Tokamak edge turbulence," IPP Report 6 333, Max-Planck-Institut fur Plasmaphysik (1999).

${ }^{3}$ B. Scott, Plasma Phys. Controlled Fusion 39, 1635 (1997).

${ }^{4}$ D. Ryutov and R. Cohen, Contrib. Plasma Phys. 44, 168 (2004); in 9th International Workshop on Plasma Edge Theory in Fusion Devices, University of California, San Diego, San Diego, CA, 3-5 September 2003.

${ }^{5}$ J. R. Myra, D. A. D'Ippolito, X. Q. Xu, and R. H. Cohen, Phys. Plasmas 7, $4622(2000)$.

${ }^{6}$ J. W. Connor, R. J. Hastie, H. R. Wilson, and R. L. Miller, Phys. Plasmas 5, 2687 (1998).

${ }^{7}$ B. LaBombard, J. Hughes, D. Mossessian, M. Greenwald, B. Lipschultz, J. Terry, and Alcator C-Mod Team, Nucl. Fusion 45, 1658 (2005).

${ }^{8}$ B. D. Scott, Phys. Plasmas 12, 062314 (2005).

${ }^{9}$ A. Zeiler, D. Biskamp, J. F. Drake, and P. N. Guzdar, Phys. Plasmas 3, 2951 (1996).

${ }^{10}$ A. Zeiler, D. Biskamp, and J. F. Drake, Phys. Plasmas 3, 3947 (1996).

${ }^{11}$ J. F. Drake, A. Zeiler, and D. Biskamp, Phys. Rev. Lett. 75, 4222 (1995).

${ }^{12}$ B. N. Rogers, J. F. Drake, and A. Zeiler, Phys. Rev. Lett. 81, 4396 (1998).

${ }^{13}$ J. W. Connor, R. J. Hastie, and J. B. Taylor, Phys. Rev. Lett. 40, 396 (1978).

${ }^{14}$ G. Bateman and D. B. Nelson, Phys. Rev. Lett. 41, 1804 (1978).

${ }^{15}$ D. R. McCarthy, P. N. Guzdar, J. F. Drake, J. T. M. Antonsen, and A. B. Hassam, Phys. Fluids B 4, 1846 (1992).

${ }^{16} \mathrm{~K}$. Mima and A. Hasegawa, Phys. Fluids 21, 81 (1978).

${ }^{17}$ M. Wakatani and A. Hasegawa, Phys. Fluids 27, 611 (1984).

${ }^{18}$ H. Sugama, M. Wakatani, and A. Hasegawa, Phys. Fluids 31, 1601 (1988).

${ }^{19}$ S. V. Novakovskii, P. N. Guzdar, J. F. Drake, C. S. Liu, and F. L. Waelbroeck, Phys. Plasmas 2, 781 (1995).

${ }^{20}$ T. Rafiq, G. Bateman, A. H. Kritz, and A. Y. Pankin, Phys. Plasmas 17, 082511 (2010).

${ }^{21}$ T. Rafiq, C. C. Hegna, J. D. Callen, and A. H. Kritz, Phys. Plasmas 16, 102505 (2009).

${ }^{22}$ A. Zeiler, D. Biskamp, J. Drake, and B. Rogers, Phys. Plasmas 5, 2654 (1998).

${ }^{23}$ A. Kendl and B. D. Scott, Phys. Rev. Lett. 90, 035006 (2003).

${ }^{24}$ P. Ricci and B. N. Rogers, Phys. Plasmas 16, 092307 (2009).

${ }^{25}$ P. Ricci and B. N. Rogers, Phys. Rev. Lett. 104, 145001 (2010).

${ }^{26}$ W. Horton, Rev. Mod. Phys. 71, 735 (1999).

${ }^{27}$ P. H. Diamond, A. Hasegawa, and K. Mima, Plasma Phys. Controlled Fusion 53, 124001 (2011).

${ }^{28}$ A. Mosetto, F. D. Halpern, S. Jolliet, and P. Ricci, Phys. Plasmas 19, 112103 (2012)

${ }^{29}$ P. Ricci and B. N. Rogers, Phys. Plasmas 20, 010702 (2013).

${ }^{30}$ F. D. Halpern, S. Jolliet, J. Loizu, A. Mosetto, and P. Ricci, Phys. Plasmas 20, 052306 (2013).

${ }^{31}$ P. Ricci, F. D. Halpern, S. Jolliet, J. Loizu, A. Mosetto, A. Fasoli, I. Furno, and C. Theiler, Plasma Phys. Controlled Fusion 54, 124047 (2012).

${ }^{32}$ J. Loizu, P. Ricci, F. D. Halpern, and S. Jolliet, Phys. Plasmas 19, 122307 (2012).

${ }^{33}$ A. Zeiler, J. Drake, and B. Rogers, Phys. Plasmas 4, 2134 (1997).

${ }^{34}$ X. Lapillonne, S. Brunner, T. Dannert, S. Jolliet, A. Marinoni, L. Villard, T. Gorler, F. Jenko, and F. Merz, Phys. Plasmas 16, 032308 (2009).

${ }^{35}$ D. A. Russell, J. R. Myra, and D. A. D'Ippolito, Phys. Plasmas 16, 122304 (2009).

${ }^{36}$ B. N. Rogers and W. Dorland, Phys. Plasmas 12, 062511 (2005).

${ }^{37}$ P. Ricci, B. N. Rogers, and S. Brunner, Phys. Rev. Lett. 100, 225002 (2008).

${ }^{38}$ F. D. Halpern, S. Jolliet, J. Loizu, A. Mosetto, and P. Ricci, Nucl. Fusion (submitted).

${ }^{39} \mathrm{~S}$. Coda and TCV Team, Nucl. Fusion 51 (2010).

${ }^{40}$ B. N. Rogers and P. Ricci, Phys. Rev. Lett. 104 (2010).

${ }^{41}$ P. Ricci, C. Theiler, A. Fasoli, I. Furno, K. Gustafson, D. Iraji, and J. Loizu, Phys. Plasmas 18, 032109 (2011).

${ }^{42}$ B. Li, B. N. Rogers, P. Ricci, K. W. Gentle, and A. Bhattacharjee, Phys. Rev. E 83, 056406 (2011).

${ }^{43}$ P. Ricci, C. Theiler, A. Fasoli, I. Furno, B. Labit, S. H. Muller, M. Podesta, and F. M. Poli, Phys. Plasmas 16, 055703 (2009). 